\title{
BEST PLAY IN DOTS \& BOXES ENDGAMES
}

\author{
DANIEL ALLCOCK
}

\begin{abstract}
We give very simple algorithms for best play in the simplest kind of Dots \& Boxes endgames: those that consist entirely of loops and long chains. In every such endgame we compute the margin of victory, assuming both players maximize the numbers of boxes they capture, and specify a move that leads to that result. We improve on results by Buzzard and Ciere [5] on the same problem: our algorithms examine only the current position and do not need to consider the game tree at all.
\end{abstract}

\section{INTRODUCTION}

Dots \& Boxes is one of the few pen-and-paper games that has a rich mathematical theory and is also played at a high level by many people without any special interest in mathematics. There are several websites where one can play online against other people, such as littlegolem.net and yourturnmyturn.com. Go shares these properties, but mathematics has more limited application in actual play [4].

We give the rules and other background in section 2. In brief, one begins with a grid of dots. When it is your turn, you join two adjacent dots. If you complete a box this way then you move again immediately. You continue in this way until you move without completing a box (when play passes to your opponent) or you complete the last box (when the game ends). Whoever completes more boxes wins. See section 2 for details and for some standard elements of play like loops and long chains, the parity rule and the hard-hearted handout. For further background we recommend [1] or [3].

The first contribution of mathematics to Dots \& Boxes is the idea of moving as though playing a simpler game called Nimstring. The rules are the same except for who wins: in Nimstring, the winner is whoever completes the last box. This sounds like a radical change, but good Nimstring moves are often good Dots \& Boxes moves. As the name suggests, Nimstring is amenable to the Sprague-Grundy theory

Date: November 26, 2018.

2010 Mathematics Subject Classification. 91A46.

Supported by Simons Foundation Collaboration Grant 429818 . 
for Nim-like games [3, Ch. 16]. This leads to a trick called the parity rule. If neither player knows it then the game proceeds mostly randomly; if only one knows it then she will win; if both know it then the game becomes challenging and interesting. The interest and challenge come from understanding the positions where there is a winning move, even when the Nimstring perspective suggests the game is lost.

An expert player who expects to lose the Nimstring game will try to steer the game toward such a position. Typically he does this by aiming for an endgame with many 3-chain and 4- and 6-loops. Therefore highlevel play requires an understanding of such positions. This paper gives a complete analysis of endgames that consist entirely of loops and long chains. We restrict attention to such positions unless otherwise indicated. We were inspired by the study of these endgames by Buzzard and Ciere [5. While their algorithms for determining the value of a game and an optimal move require only linear time, they involve trees of cases and subcases, and also a limited form of recursion. Our algorithms are simpler.

If it is your turn and you must choose a loop or long chain to open (play in) then we call you the opener. Once you choose this component $C$, it does not matter where in $C$ you play. Your opponent will either take all those boxes, or reply with the hard-hearted handout (see below), and where you play in $C$ has no effect on these options.

Nimstring suggests that you have lost. We will see that you cannot win the endgame, in the sense that you cannot capture more of the remaining boxes than a skilled opponent. But if you captured more boxes than she did, before reaching the endgame, and you lose the endgame by only a little, then you may still be able to win. The strategy in theorem 1.1 is guaranteed to lose the endgame by as little as possible, and therefore gives your best chance of winning. Your opponent is called the controller, for reasons explained below.

A chain or loop of length $N$ is called an $N$-chain or $N$-loop, and often indicated by simply writing $N$, attaching a subscript $\ell$ to indicate a loop. We use additive notation in the obvious way, for example $G=3+4_{\ell}+8_{\ell}$ means that $G$ consists of a 3-chain, a 4-loop, an 8-loop and possibly some already-claimed boxes:

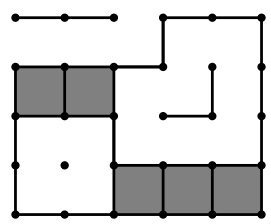


In actual play the claimed boxes (shaded here) would be marked with the players' initials. We ignore such boxes when discussing $G$ because they do not affect play. The value $v(G)$ of any endgame $G$ means the margin by which the controller will beat the opener, assuming that they enter the endgame with a tie score and then play optimally. So $v\left(3+4_{\ell}+8_{\ell}\right)=1$ means that the opener can win if and only if he earned at least a 2-box advantage during whatever play led to this endgame. To lose this endgame by only one box, open the 4-loop first and the 8-loop second.

The size of $G$ means the number of (unclaimed) boxes in $G$, and the controlled value $c(G)$ is defined below and explained in section 2, For now it is enough to know that you can compute $c(G)$ quickly in your head. The standard move means to open a 3-chain if one is present, otherwise a shortest loop if a loop is present, and otherwise a shortest chain. See section 3 for the proof of the following result.

Theorem 1.1 (Opener strategy). Suppose $G$ is a nonempty Dots 86 Boxes position that consists of loops and long chains. In each of the following cases, opening the shortest loop is optimal:

(1) $c(G) \geq 2 \quad$ and $G=3+$ (one or more loops);

(2) $c(G) \in\{0, \pm 1\}$ and $G=4_{\ell}+$ (anything except $3+3+3$ );

(3) $c(G) \leq-2$ and $G=4_{\ell}+3+H$, where $4 \mid \operatorname{size}(H)$ and $H$ has no 3-chains.

In all other cases the standard move is optimal.

The "phase transition" when $c(G)$ changes from 1 to 2 was discovered by Berlekamp. The transition from -2 to -1 appears to be new. The proofs of this theorem and those below involve much case analysis, but the results can be assembled afterwards into fairly simple conclusions.

Berlekamp introduced the controlled value $c(G)$ because it is easy to compute and carries a lot of information about $v(G)$. For example, they are equal if $c(G) \geq 2$ (see theorem 3.2). The definition is

$$
c(G)=\operatorname{size}(G)-4(\# \text { long chains })-8(\# \text { loops })+\operatorname{tb}(G)
$$

where $\operatorname{tb}(G)$ is called the terminal bonus of $G$ and defined by

$$
\operatorname{tb}(G)= \begin{cases}0 & \text { if } G \text { is empty } \\ 8 & \text { if } G=\text { (one or more loops) } \\ 6 & \text { if } G=\text { (one or more loops) }+ \text { (one or more 3-chains) } \\ 4 & \text { otherwise }\end{cases}
$$

See section 2 for why $c(G)$ is called the controlled value. 
Example 1.2. Suppose $G$ consists of five 3-chains, a 4-loop and an 8loop. We have $c(G)=27-4 \cdot 5-8 \cdot 2+6=-3$. The theorem says that opening the components in the order $3,3,4_{\ell}, 3,3,8_{\ell}, 3$ is optimal. We used a computer to check this, and also that the only other optimal line of play is $3,4_{\ell}, 3,3,3,8_{\ell}, 3$. This shows that there is a certain subtlety to the order in which one must open the components, and suggests that theorem 1.1 may be the simplest strategy possible.

If you are the controller, and the opener has just opened a loop or long chain, then the decision you face is whether to keep control or give it up. Giving up control means that you take all of the boxes in the just-opened component. Unless that ends the game you must move again, so you become the opener and your opponent the controller. As the new opener, you can use theorem 1.1 to choose which component to open. Keeping control means that you take all but a few of the boxes in the opened component (4 for a loop or 2 for a long chain). This is called the hard-hearted handout: a handout because you are giving your opponent some boxes, and hard-hearted because after he takes them he must open the next component. In this case he remains the opener and you remain the controller.

Now we consider the situation where the opener has just opened component $C$ of a position $G$. It is easy to see that the controller should keep control if $v(G-C)$ is larger than the number of boxes (2 or 4) given away in the hard-hearted handout, and give up control if $v(G-C)$ is smaller. She may choose either option in case of equality. We use subtractive notation in the obvious way: $G-C$ means the position got from $G$ by removing $C$. So the following well-known result gives an optimal strategy for the controller.

Theorem 1.3 (Controller strategy). If the opener has just opened a component $C$ of $G$, then the following gives an optimal move for the controller. Keep control if $C$ is a loop and $c(G-C)>4$, or if $C$ is chain and $v(G-C)>2$; otherwise give up control.

To use this strategy the controller must be able to recognize when $v(G-C)>2$ or 4 . It is very easy to recognize when $v(G-C)>4$ because this is equivalent to $c(G-C)>4$. This and the following result are corollaries of theorem 4.1, which gives all all values explicitly.

Theorem 1.4 (Values $>2$ ). We have $v(G)>2$ if and only if: either $c(G)>2$, or else $G$ satisfies one of the two alternatives

$G$ has exactly one 3 -chain and $\operatorname{size}(G) \equiv 3 \bmod 4$

$G$ has no 3-chains and $\operatorname{size}(G) \not \equiv 2 \bmod 4$

and one of the two alternatives 


$$
\begin{aligned}
& c(G)+4 f(G)>2 \text { and } c(G) \equiv \pm 3 \text { or } 4 \bmod 8 \\
& c(G)+4 f(G)<2 \text { and } f(G) \text { is even }
\end{aligned}
$$

where $f(G)$ is the number of 4-loops in $G$.

In particular, if two or more 3-chains are present then $v(G)>2$ if and only if $c(G)>2$. The same holds if just one is present and $\operatorname{size}(G) \not \equiv 3 \bmod 4$. In the remaining cases we prefer theorem 4.2(3) to the complicated second pair of alternatives. It gives $v(G)$ as the result of a simple process applied to a simple starting number, and is much easier to remember.

Section 5 gives some consequences for mid-game strategy. For example, the player who is opener when the endgame begins will probably lose if he has only a 1-box advantage, but very likely win with a 2-box advantage. This assumes an odd $\times$ odd board and that enough 3 -chains and 4- and 6-loops are present to make $c(G)<2$. Players often sacrifice a box or two during the midgame to create the "right" number of long chains. (The goal is to be controller in the endgame, because in Nimstring the controller always wins. See the parity rule in section 2.) To first approximation our results show that sacrificing one box is safe but sacrificing two is suicide.

The natural next endgames to consider have components more complicated than loops and chains, such as

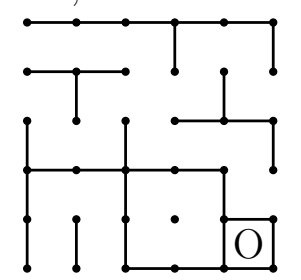

In this example every move is loony (ie, loses the Nimstring game), but if the controller always keeps control then the opener O can win. She starts this endgame with a 1-box advantage and can choose to play as though in $4_{\ell}+4+4+4+8$, which has value 0 . One could also allow loops to have odd length. It is conceivable that our strategy for the opener remains valid. But length 7 loops definitely do complicate some intermediate results, as in [5, remark 16], and length 5 loops are probably worse.

We are grateful to Kevin Buzzard and Michael Burton for interesting and helpful conversations. In particular, this paper would not exist without the prior work of Buzzard and Ciere [5]. We also remark that William Fraser announced on the games website littlegolem. net that on April 28, 2017 he finished the calculations needed for his program 
The Shark to play the $5 \times 5$ game perfectly. One can play against it on this website, but no further details seem to be public.

\section{Dots \& Boxes}

In the first part of this section we review the rules of Dots \& Boxes and some standard elements of good play. The main references are 3 , Ch. 16] and [1]. In the second part we discuss some more-technical material that we will need.

The game begins with a grid of dots. At the end of the game, each pair of (horizontally or vertically) adjacent dots will be joined by an edge, making a grid of boxes. The board size is typically $5 \times 5$, meaning 5 boxes by 5 boxes. Odd $\times$ odd boards are best because ties are impossible. After the game, each box will belong to one player or the other, and whoever has the most boxes will win. On her turn, a player moves by drawing a line connecting two adjacent dots that are not yet joined. Any boxes completed by this line then belong to her, and if at least one box was completed then she moves again immediately. She continues in this way until she moves without completing a box (when it becomes the other player's turn) or she completes the grid (ending the game). In particular, a player's turn may consist of more than one move. It often happens that the segment completing a box also completes the third edge of another box. In this case the extra move earned by completing the first box enables her to complete the second box, which in turn might allow her to complete a third box, and so on. In this way a player may capture an entire chain of boxes in a single turn.

A sample position appears in figure1. During earlier play the author completed three boxes and placed his initial inside them to mark them as his. This makes it looks like he is winning, but it is his turn and no good move is available. Any move in the loop on the right lets his opponent C (for "controller") capture all 10 boxes there. Similarly, any move in the chain on the left lets her capture all 12 of its boxes. When a child, the author would have moved in the loop, expecting her to capture those 10 boxes and then open the 12-chain for him to capture. He would win, 15-10.

Unfortunately for the author, $\mathrm{C}$ knows the hard-hearted handout: when he offers her the 10 boxes, she takes all but 4, for example as shown in the top line of play. Dotted lines indicate her moves. Note the $2 \times 1$ and $1 \times 2$ rectangles that she could have claimed but chose not to. Your poor author wins those 4 boxes but then must move again, opening the 12-chain for $\mathrm{C}$ to capture entire. Therefore $\mathrm{C}$ wins, 18-7. 


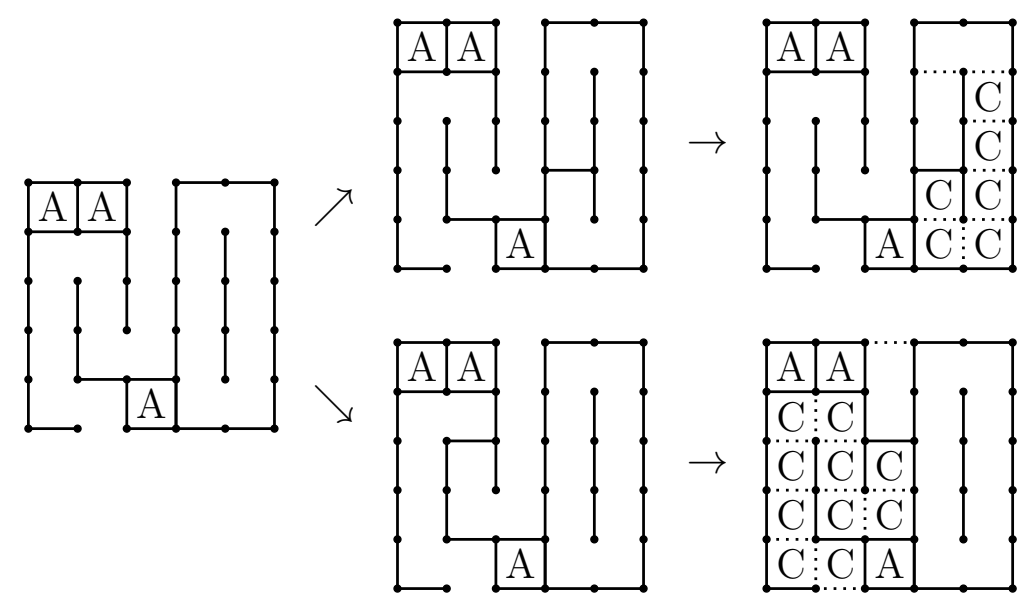

Figure 1. A Dots \& Boxes position consisting of a 12chain and a 10-loop, and two lines of play demonstrating the hard-hearted handout

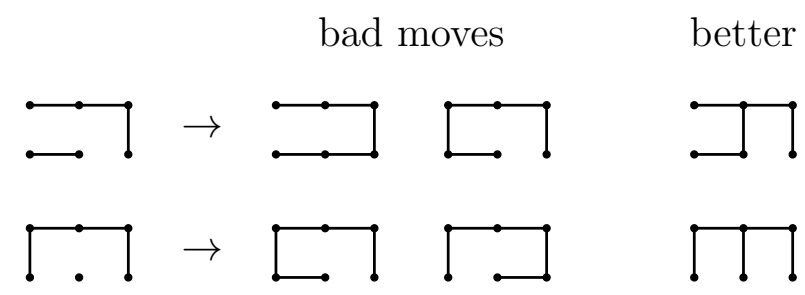

Figure 2. Opening a 2-chain in one of the "bad" ways allows the opponent to choose between capturing both boxes and then moving again, or replying with the hardhearted handout. Bisecting the 2-chain as shown on the right removes the opponent's second option.

If the author opens the chain instead of the loop then $\mathrm{C}$ takes all but two of its boxes, as shown in the bottom line of play. This is the chain version of the hard-hearted handout. In this case the author would lose 20-5. (A few people play with a rule that forbids the hard-hearted handout: if one can complete a box then one must. But forced greedy strategies make games dull.)

A chain of length 2 does not present the same problem, because if you split it into two single boxes then your opponent has no choice but to open the next component. Two examples appear in figure 2. The "long" in "long chain" restricts attention to chains of length 3 or more. In this paper we are restricting attention to positions consisting of loops and long chains, so chains of length 2 will not appear. So henceforth 
we will write "chain" for long chain, sometimes saying "long chain" just for emphasis.

If $\mathrm{C}$ always used the hard-hearted handout, then she would capture all but 2 from each chain and all but 4 from each loop, except that she would capture all of the last component. If the loops and chains are not too small then this leads to very lopsided scores. To first approximation the game is always lost by whoever has to open the first loop or long chain. This is the connection between Dots \& Boxes and Nimstring, and leads to the long chain rule [3, Ch. 16]. This rule predicts that the first resp. second player will win if there are an even resp. odd number of long chains in the endgame (assuming an odd $\times$ odd board). So in advanced play, the early game and midgame are all about trying to create chains, or obstruct their creation, so that the desired number $(\bmod 2)$ are formed.

As in the introduction, we call the two players the opener and controller. Which player is which can change during a game. Whoever has no choice but to open some chain or loop is the opener, and whoever replies to a just-opened chain or loop is the controller. In figure 1 the author was the opener and his opponent the controller. But sometimes the controller should give up control by taking all of an opened chain or loop. By doing this she becomes the opener and her opponent becomes the controller.

For example, if the position consists of $N>1$ chains of length 3 , one of which was just opened, then a controller who keeps control except at the end will give her opponent 2 boxes from each chain but the last. She scores $(N-1)+3$ to her opponent's $2(N-1)$, so she loses if $N>4$. If $N>3$ then it is better to take all 3 boxes of the opened chain. Being forced to move again, she is now the opener: she opens a chain and her opponent (now the controller) faces a similar decision. In one line of optimal play, the players take turns giving up control, except that whoever responds in the second-to-last chain keeps control, giving up 2 boxes there but getting all 3 in the last chain. If $N$ is even then the original controller will be the one to do this, and wins $\frac{3}{2} N+1$ to $\frac{3}{2} N-1$. When $N$ is odd she does not get to do this, but still wins $\frac{3}{2} N+\frac{1}{2}$ to $\frac{3}{2} N-\frac{1}{2}$.

This article is about deciding what to open when you must open something, and deciding whether to keep control when you have it.

The rest of this section is more technical. We have said that we will consider only Dots \& Boxes positions $G$ consisting of loops and long chains, where long means of length $\geq 3$. This is not literally true in two cases. Immediately after the opener has opened a component $C$, and 
whenever the controller keeps control, one or two opened components are present. But we will be able to phrase all our analysis in terms of $G-C$. So we adopt the convention that unless opened components are specifically allowed, Dots \& Boxes positions consist of unopened loops and long chains. We also note that the length of a loop must be even and at least 4 , by the geometry of the grid.

If $G$ is a Dots \& Boxes position then we define

$\begin{aligned} \operatorname{size}(G) & \text { number of boxes not yet claimed } \\ \theta(G) & \text { number of 3-chains } \\ f(G) & \text { number of 4-loops } \\ s(G) & \text { number of 6-loops } \\ v(G) & \text { value see section } 1 \text { and below } \\ c(G) & \text { controlled value see }(1.1) \text { and below } \\ \operatorname{tb}(G) & \text { terminal bonus - see } 1.2\end{aligned}$

Recall that when discussing $G$ we ignore any boxes captured earlier in the game, so $\operatorname{size}(G)$ is not the total number of boxes in the grid, but the number of unclaimed boxes. We call $G$ even or odd according to whether $\operatorname{size}(G)$ is even or odd. Usually the symbol $G$ will represent whatever position is of central interest, so we will use the abbreviations $\theta, f, s, v$ and $c$ for $\theta(G), f(G), s(G), v(G)$ and $c(G)$. We will still use functional notation for other positions, for example $\theta(G+3)=\theta+1$.

We recall the meaning of the value $v(G)$ from section 1 ; the opener will lose by $v(G)$ boxes if both players maximize the number of boxes they take. The trivial example is that $v(G)=\operatorname{size}(G)$ when $G$ consists of a single component. After the opener opens it, the controller "gives up control" by claiming all the boxes and ending the game. The next example is $v(3+3)=2$. The opener opens a chain, the controller keeps control (replying with the hard-hearted handout). The opener accepts the 2 boxes in the handout and opens the other chain, all of which the controller claims. So $v(3+3)=4-2=2$.

Once the opener has opened a component $C$, the controller has really only two choices. First, he may keep control and then play optimally, in which case he will finish the game with

$$
\begin{array}{ll}
(\operatorname{size}(C)-4)+v(G-C) & \text { if } C \text { is a chain } \\
(\operatorname{size}(C)-8)+v(G-C) & \text { if } C \text { is a loop }
\end{array}
$$

more boxes than his opponent. Second, he may give up control and then play optimally, in which case he will win by $\operatorname{size}(C)-v(G-C)$. The minus sign appears because when the controller gives up control, he enters the position $G-C$ as the opener, so his opponent is the one who will score $v(G-C)$. We write $v(G ; C)$ for the higher of these two margins of victory. One should think of this as the value of $G$, given 
that the opener has just opened $C$. Obviously the controller will choose the higher-scoring option, leading to

$$
v(G ; C)= \begin{cases}(\operatorname{size}(C)-2)+|v(G-C)-2| & \text { if } C \text { is a chain } \\ (\operatorname{size}(C)-4)+|v(G-C)-4| & \text { if } C \text { is a loop }\end{cases}
$$

We will use this repeatedly without specific reference. Another way to express this is that if $v(G-C)=2$ resp. 4 and $C$ is a chain resp. loop, then keeping and giving up control are equally good options. If $v(G-C)$ is more than this then the controller should keep control, and if it is less then he should give up control.

Now consider the opener's perspective, facing a nonempty position $G$. He will obviously prefer to open whichever component $C$ minimizes $v(G ; C)$. So

$$
v(G)=\min _{C} v(G ; C)
$$

where $C$ varies over the components of $G$. The previous paragraph shows that each $v(G ; C)$ is nonnegative, which proves $v(G) \geq 0$. This justifies our assertion that the opener can never win the endgame. Also, combining (2.2) and the previous paragraph gives a way to evaluate $v(G)$ recursively. As an example we work out how to play the position $G=3^{n}$, meaning that $G$ consists of $n$ many 3 -chains. The opener has no real choice about which component to open, so $v\left(3^{n}\right)=$ $v\left(3^{n} ; 3\right)=1+\left|v\left(3^{n-1}\right)-2\right|$ whenever $n>0$. Induction gives $v\left(3^{n}\right)=$ $0,3,2,1,2,1,2,1, \ldots$ when $n=0,1,2,3,4,5,6,7, \ldots$ So the controller must keep control if $n=2$, and must give up control if either $n=1$ or $n$ is even and larger than 2. In all other cases the choice of keeping or giving up control makes no difference. The simplest rule is to keep control only when $n=2$.

Now we can explain the controlled value $c(G)$, defined in 1.1 . Another useful formula for it is

$$
c(G)=\sum_{i}\left(c_{i}-4\right)+\sum_{j}\left(l_{j}-8\right)+\operatorname{tb}(G)
$$

where $c_{1}, c_{2}, \ldots$ are the lengths of the chains, $l_{1}, l_{2}, \ldots$ are the lengths of the loops, and the terminal bonus was defined in (1.2). Berlekamp introduced the idea of a controller who follows the control strategy of always keeping control, except in a few cases when he obviously shouldn't. In our formulation, the controlled value is the margin by which a controller publicly committed to this strategy will win the endgame $G$ against a skilled opener. The role of the public commitment 
is to simplify the analysis of his opponent's strategy. As usual, $c(G)<0$ indicates a loss for the controller not a win.

Our formulation of the control strategy is: keep control until the opener opens the last component, or opens a loop and only 3-chains remain. In these cases, give up control. In the second case, play continues after giving up control, the player is now the opener, and only 3-chains remain. He opens them until he regains control, if he ever does, when he returns to the control strategy.

Our control strategy differs from the one in [1, p. 84] in how the controller plays if he gives up and then later recovers control. Although we don't need it, we remark that this change doesn't affect the final score. See lemma 13 in [5] for the analogue of our next result.

Theorem 2.1 (Controlled value). Suppose $G$ is a Dots 6 Boxes position consisting of loops and long chains. If the controller follows the control strategy, and the opener knows this and plays optimally, then the controller will win by $c(G)$.

Proof. Because the roles of controller and opener can change during play, we write $K$ resp. $O$ for the player who starts out as the controller resp. opener. We write $d(G)$ for the amount by which $K$ will win, assuming best play by $O$. We must show $d(G)=c(G)$. If $C$ is a component then we write $d(G ; C)$ for what the final score would be if $O$ opens $C$ and then plays optimally. Obviously $d(G)=\min _{C} d(G ; C)$.

We induct on the number of components. The base case is that $G$ has one component (or none). Then $c=\operatorname{size}(G)=v$. For the inductive step, suppose first that $G$ does not consist of a loop and (one or more) 3 -chains. After $O$ opens component $C, K$ will keep control, so

$$
\begin{aligned}
d(G ; C) & =\operatorname{size}(G-C)-\left(\begin{array}{l}
4 \text { if } C \text { is a chain } \\
8 \text { if } C \text { is a loop }
\end{array}\right)+d(G-C) \\
& =c(G)-\operatorname{tb}(G)+\operatorname{tb}(G-C)
\end{aligned}
$$

We have used the inductive hypothesis $d(G-C)=c(G-C)$ and applied (1.1) to both $G-C$ and $G$. This shows that $O$ should open the component $C$ that minimizes $\operatorname{tb}(G-C)$. If $C$ is a loop then removing it leaves the terminal bonus invariant. (The only way it could change is if it were the last loop and only 3-chains remain, but we have set that case aside.) If $C$ is a chain then removing it increases the terminal bonus or leaves it the same. Therefore, if there is a loop then $O$ should choose it as $C$, and

$$
d(G)=d(G ; C)=c(G)-\operatorname{tb}(G)+\operatorname{tb}(G-C)=c(G)
$$


If there is no loop then the removal of any chain leaves the terminal bonus invariant, and the same calculation shows $d(G)=c(G)$.

It remains to consider the case that $G$ consists of a loop $L$ and $n \geq 1$ many 3 -chains. We write $l$ for the length of the loop. Our argument for $d(G ; C)$ still applies if $C=3$, namely

$$
d(G ; 3)=c(G)-\operatorname{tb}(G)+\operatorname{tb}(G-3)= \begin{cases}l-1 & \text { if } n=1 \\ l-n-2 & \text { if } n>1\end{cases}
$$

The key point is to compute $d(G ; L)$. If $O$ opens $L$, then $K$ will give up control, and then $O$ may keep control for however many turns $m \leq n$ he desires. In fact $O$ will choose $m<n$ because giving away two boxes of the last component would be silly. After $O$ gives up control, $K$ will keep it until the last chain. This leads to

$$
\text { final score }= \begin{cases}l+n-4 & \text { if } m=n-1 \\ l+2 m-n-2 & \text { if } 0 \leq m<n-1\end{cases}
$$

If $n>1$ then all these values are $\geq d(G ; 3)$, so $O$ should open the 3 chain and $d(G)=d(G ; 3)=c(G)$. If $n=1$ then $m$ must be $0=n-1$, so $d(G ; L)=l-3$. Since this is less than $v(G ; 3)=l-1, O$ should open the loop. And $d(G)=d(G ; L)=l-3=c(G)$.

We close this section with some relations between the various quantities we have introduced.

Lemma 2.2. Suppose a Dots $\&$ Boxes position $G$ consists of loops and long chains. Then

(1) $c(G) \leq v(G)$;

(2) $\operatorname{size}(G) \equiv c(G) \equiv v(G) \equiv v(G ; C)$ mod 2 , for every component $C$ of $G$;

(3) $\operatorname{size}(G) \equiv c(G) \bmod 4$ if $G$ has no 3-chains.

We also mention two more relations that we will prove later. First, adding the hypothesis that $G$ is even to part (3) strengthens its conclusion to $v(G) \equiv \operatorname{size}(G) \equiv c(G) \bmod 4$. (See theorem 4.1.) Second, our theorem 3.2 contains Berlekamp's result $c(G) \geq 2 \Longrightarrow c(G)=v(G)$.

Proof. (1) The controller may guarantee a final score of at least $c(G)$ by publicly committing to the control strategy.

(2) Both $c(G)$ and $v(G)$ have the same parity as $\operatorname{size}(G)$ because they are margins of victory under certain lines of play. For the last congruence, it follows from (2.1) that $v(G ; C)$ has the same or different parity as $v(G-C)$ according to whether size $(C)$ is even or odd. Also, $\operatorname{size}(G)$ has the same or different parity as $\operatorname{size}(G-C)$ under the same 
conditions. Since size $(G-C)$ and $v(G-C)$ have the same parity, it follows that $v(G ; C) \equiv \operatorname{size}(G) \bmod 2$.

(3) When no 3-chains are present the terminal bonus is divisible by 4 . So our claim follows from the definition 1.1 of $c(G)$.

\section{Opener StRAtegy}

Our goal in this section is to prove theorem 1.1, under the standing hypothesis is that $G$ is a Dots \& Boxes position consisting of unopened loops and long chains. Although our proof is logically independent of the work of Buzzard and Ciere [5], and organized very differently, we would not have been able to formulate theorem 1.1 or the lemmas below without reference to it. We regret that we do not use their lovely man-in-the-middle and chain- and loop-amalgamation arguments.

Lemma 3.1 (Tiny positions). Suppose $G$ has one component, or consists of a 3-chain and a loop. Then $v(G)=c(G)$. In the second case, opening the loop is optimal.

Proof. The first case is obvious. For the second case one examines the four possible lines of play. (Opening the 3-chain is not optimal.)

The next theorem summarizes the key points of the lemma after it. After establishing it we will use the implication $(c \geq 2) \Longrightarrow(v=c)$ many times without specific reference. The lemma and its proof are essentially the same as lemma 15 from [5], that one can "fly the plane without crashing" in the sense of [2].

Theorem 3.2 (Large controlled values). Suppose $c(G) \geq 2$. Then $v(G)=c(G)$ and the following gives an optimal move:

(1) Open a 3-chain (if $G$ has one and at least one other chain).

(2) Otherwise, open a shortest loop (if $G$ has a loop).

(3) Otherwise, open a shortest chain.

Lemma 3.3 (Large controlled values-details). Suppose $c(G) \geq 2$.

(1) If $C$ is any component satisfying

$$
\operatorname{tb}(G-C)=\operatorname{tb}(G) \text { and } c(G-C) \geq \begin{cases}2 & \text { if } C \text { is a cycle } \\ 4 & \text { if } C \text { is a loop }\end{cases}
$$

then opening $C$ is optimal.

(2) If $G$ has a 3-chain, 4-loop or 6-loop, whose removal does not alter the terminal bonus, then opening it is optimal.

(3) Suppose G has no 3-chains, or exactly one 3-chain and no other chains. If $G$ has a loop then opening a shortest loop is optimal. 
(4) If $G$ has no loops, then opening a shortest chain is optimal.

(5) $v(G)=c(G)$.

Proof. We induct on the number of components. If $G$ has one component then (1)-(2) are vacuous and (3)-(5) are trivial. So suppose $G$ has at least two components.

(1) Write $l$ for the length of $C$, and assume first that $C$ is a loop. We have

$$
v(G ; C)=(l-4)+|v(G-C)-4|
$$

Since $c(G-C) \geq 4$ by the hypothesis of (1), induction gives $v(G-C)=$ $c(G-C) \geq 4$. So we have

$$
v(G ; C)=(l-4)+c(G-C)-4=(l-8)+c(G-C)
$$

Since $G$ and $G-C$ have the same terminal bonus, $c(G-C)=c(G)-$ $(l-8)$. Plugging this in gives $v(G ; C)=c(G)$. Together with lemma 2.2 this gives

$$
c(G) \leq v(G) \leq v(G ; C)=c(G)
$$

Therefore $v(G ; C)=v(G)$, which proves optimality, and $v(G)=c(G)$. If $C$ is a chain then the argument is the same with all 4's replaced by 2's and all 8's by 4's.

(2) First suppose $G$ has a 3-chain with $\operatorname{tb}(G-3)=\operatorname{tb}(G)$. Then $c(G-3)=1+c(G) \geq 3$. So (1) applies to the 3-chain. And similarly for a 4-loop or 6-loop.

(3) By hypothesis, $G$ consists of either at least one loop and a 3chain, or at least one loop and possibly some chains of length $\geq 4$. Let $C$ be a shortest loop. In the special case $G=C+3$, opening $C$ is optimal by lemma 3.1. So suppose otherwise: $G$ has a second loop, or a chain of length $\geq 4$. In either case the removal of $C$ leaves the terminal bonus invariant. If $C=4_{\ell}$ or $6_{\ell}$ then we are done by (2). There are two remaining cases. First, $G$ consists of a 3 -chain and two or more loops of length $\geq 8$. Second, $G$ consists of at least one loop of length $\geq 8$ and possibly some chains of length $\geq 4$. In each case we write out formula (2.3) for $c(G-C)$ and use the absence of 4 - and 6 -loops. The results in the two cases are

$$
\begin{aligned}
c(G-C) & =(3-4)+(\text { nonnegative terms })+6 \geq 5 \\
\text { and } \quad c(G-C) & =(\text { nonnegative terms })+(4 \text { or } 8) \geq 4
\end{aligned}
$$

So (1) shows that opening $C$ is optimal.

(4) $G$ consists of at least 2 chains, so removing any one of them leaves the terminal bonus unchanged. Let $C$ be shortest possible. If it is a 
3-chain then we appeal to (2). Otherwise we mimic the argument at the end of the proof of (3):

$$
c(G-C)=4+(\text { nonnegative terms }) \geq 4
$$

Since the right side is at least 2 , it follows from (1) that opening $C$ is optimal.

(5) One of (2)-(4) applies to $G$. Their proofs show that either $G$ consists of a 3 -chain and a loop, or else $G$ has a component $C$ satisfying (1). In the first case we appeal to lemma 3.1 for the equality $v=c$. In the second case we observed $v=c$ during the proof of (1).

Lemma 3.4 (Example of $6_{\ell^{-o p t i m a l i t y}}$ ). Suppose $G \neq \emptyset$ has no 3chains or 4-loops, and $c(G) \leq 4$. Then

$$
v(G)=\left\{\begin{array}{cl}
3 & \text { if } \operatorname{size}(G) \text { is odd } \\
\text { (whichever of } 2,4) \equiv \operatorname{size}(G) \bmod 4 & \text { otherwise }
\end{array}\right.
$$

Furthermore, if $c(G)<2$ then $G$ has at least two 6-loops and opening one of them is optimal.

Proof. We write $w(\cdot)$ for the function on positions defined by the formula. First we establish the cases $c=2,3,4$. By the absence of 3-chains, lemma 2.2 (3) gives $\operatorname{size}(G) \equiv c \bmod 4$. So the definition of $w$ gives $w(G)=c$. And theorem 3.2 gives $v=c$, completing the proof when $c \geq 2$.

So we may suppose $2>c$, which we write out using (2.3)

$$
2>c=-\theta-4 f-2 s+\text { (nonnegative terms) }+\operatorname{tb}(G)
$$

By $\operatorname{tb}(G) \geq 4$ and $\theta=f=0$ we get $s \geq 2$. Since there are at least two loops, the removal of any loop leaves the terminal bonus unchanged. In particular, $c\left(G-6_{\ell}\right)=c+2$. Also, $G$ has a third component because $c(G)<2$ and $c\left(6_{\ell}+6_{\ell}\right)=4$. Having made these preparations, we now induct on the number of components.

First we claim $v\left(G-6_{\ell}\right)=w\left(G-6_{\ell}\right)$. If $c\left(G-6_{\ell}\right) \geq 2$ then $c\left(G-6_{\ell}\right)=$ 2 or 3 , which are cases already proven. This includes the base case that $G$ has 3 components, because then $G-C=6_{\ell}+C^{\prime}$ with $C^{\prime} \neq 3,4_{\ell}$, which forces $c(G-C) \geq 2$. On the other hand, if $c\left(G-6_{\ell}\right)<2$ then induction gives $v\left(G-6_{\ell}\right)=w\left(G-6_{\ell}\right)$.

Next we claim $v\left(G ; 6_{\ell}\right)=w(G)$. The previous paragraph gives us

$$
v\left(G ; 6_{\ell}\right)=2+\left|w\left(G-6_{\ell}\right)-4\right|= \begin{cases}3 & \text { if } \operatorname{size}\left(G-6_{\ell}\right) \text { is odd } \\ 2 & \text { if } \operatorname{size}\left(G-6_{\ell}\right) \equiv 4 \bmod 4 \\ 4 & \text { if } \operatorname{size}\left(G-6_{\ell}\right) \equiv 2 \bmod 4\end{cases}
$$


Because the sizes of $G$ and $G-6_{\ell}$ differ by $2 \bmod 4$, the right side is $w(G)$, proving the claim. Once we prove that opening a 6 -loop is optimal it will follow that $v=v\left(G ; 6_{\ell}\right)=w(G)$ and the induction will be complete.

Suppose that some component $C \neq 6_{\ell}$ has $v(G ; C)<v\left(G ; 6_{\ell}\right)$. We have

$$
2 \leq v(G ; C) \underset{\bmod 2}{\equiv} v\left(G ; 6_{\ell}\right) \leq 4
$$

The first inequality comes from (2.1) because $C$ is a chain of length $\geq 4$ or a loop of length $\geq 8$. The congruence is Lemma 2.2 (2), and we saw $v\left(G ; 6_{\ell}\right) \leq 4$ in the previous paragraph. This forces

$$
v(G ; C)=2 \text { and } v\left(G ; 6_{\ell}\right)=4 .
$$

Together with (2.1), the first shows that $C$ is a 4 -chain with $v(G-4)=$ 2. Together with (3.1), the second shows that $\operatorname{size}(G) \equiv 4 \bmod 4$.

This is impossible because $v(G-4) \equiv 4 \bmod 4$ : by induction if $c(G-4)<2$, or by

$$
v(G-4)=c(G-4) \equiv \operatorname{size}(G-4) \equiv 4 \bmod 4
$$

if $c(G-4) \geq 2$. (The first congruence is lemma 2.2.)

We will use the argument for (3.2) and (3.3) several more times, without giving the details each time.

Lemma 3.5 (First example of 3-optimality). Suppose $c(G)<2$ and that $G$ has no 4-loops, but does have a 3-chain. Then opening a 3-chain is optimal, and

$$
v(G)= \begin{cases}2 & \text { if } G \text { is even } \\ 3 & \text { if } \theta=1 \text { and } \operatorname{size}(G) \equiv 3 \bmod 4 \\ 1 & \text { otherwise }\end{cases}
$$

Proof. We write $w(\cdot)$ for this function on positions, and prove $v=c$ by induction on $\theta$. In both the base case and the inductive step the strategy is to show $v(G ; 3)=w(G)$. Given this, the optimality of a 3-chain follows from

$$
2 \leq v(G ; C) \underset{\bmod 2}{\equiv} v(G ; 3)=w(G) \leq 3
$$

for every component $C$ other than a 3-chain.

Now for the induction. By the hypothesis $c<2, G$ does not consist of a single 3-chain, so there is another component. This implies that the terminal bonus rises by at most 2 if a 3 -chain is removed. In particular, 
$c(G-3) \leq c+3 \leq 4$. If $\theta=1$ then $v(G-3)$ is given by lemma 3.4 . This yields

$$
v(G ; 3)=1+|v(G-3)-2|= \begin{cases}2 & \text { if } \operatorname{size}(G-3) \text { is odd } \\ 3 & \text { if } \operatorname{size}(G-3) \equiv 4 \bmod 4 \\ 1 & \text { if } \operatorname{size}(G-3) \equiv 2 \bmod 4\end{cases}
$$

This is visibly equal to $w(G)$. Now suppose $\theta>1$. We must show that $v(G ; 3)$ is equal to whichever of 1 and 2 has the same parity as $G$. It is enough to show $v(G ; 3) \in\{1,2\}$. In turn, this will follow once we prove $v(G-3) \in\{1,2,3\}$. We observe $c(G-3)=c+1 \leq 2$. In the case $c(G-3)=2$ we have $v(G-3)=c(G-3)=2$. In the case $c(G-3)<2$ we have $v(G-3) \in\{1,2,3\}$ by induction. This finishes the proof.

Lemma 3.6 (3's and $4 \ell_{\ell}$ 's). Suppose $G$ consists of 3-chains and 4-loops. Then

$$
v(G)=\left\{\begin{array}{cl}
(\text { whichever of } 0,4) \equiv \operatorname{size}(G) \bmod 8 & \text { if } \theta=0 \\
3 & \text { if } G=3+4_{\ell}^{\text {even }} \\
(\text { whichever of } 1,2) \equiv \operatorname{size}(G) \bmod 2 & \text { otherwise }
\end{array}\right.
$$

\section{Furthermore,}

(1) Opening a 4-loop is optimal if and only if $\theta$ is even or 1.

(2) Opening a 3-chain is optimal if and only if $\theta \geq 2$ or $f$ is even.

Proof. Each entry in the following table is the smaller of

$$
\begin{aligned}
v\left(G ; 4_{\ell}\right) & =\mid(\text { the entry to the left })-4 \mid \\
\text { and } \quad v(G ; 3) & =1+\mid(\text { the entry above })-2 \mid .
\end{aligned}
$$

Except: in the left column there is no $v\left(G ; 4_{\ell}\right)$ and in the top row there is no $v(G ; 3)$. The top left entry is $v(\emptyset)=0$. It follows by induction that the table gives $v(G)$, which justifies all our claims.

\begin{tabular}{rlllllllll}
$f=$ & 0 & 1 & 2 & 3 & 4 & 5 & 6 & $\cdots$ \\
\hline$\theta=0$ case: & $v(G)=$ & 0 & 4 & 0 & 4 & 0 & 4 & 0 & \\
$\theta=1$ case: & $v(G)=$ & 3 & 1 & 3 & 1 & 3 & 1 & 3 & \\
$\theta=2$ case: & $v(G)=$ & 2 & 2 & 2 & 2 & 2 & 2 & 2 & \\
$\theta=3$ case: & $v(G)=$ & 1 & 1 & 1 & 1 & 1 & 1 & 1 &
\end{tabular}

Starting with $\theta=2$, the rows alternate between all 2's and all 1's.

Lemma 3.7 (First example of $4_{\ell}$-optimality). Suppose $G$ has a 4 -loop and $c(G) \geq-2$. Then

(1) $v(G)=|c(G)|$ unless $G=3+3+4 \ell$, in which case $v(G)=2$.

(2) Opening a 4-loop is optimal unless $G=3+3+3+4_{\ell}$. 
Proof. First we treat the case that removing a 4-loop changes the terminal bonus. This can only happen when $G$ consists of a 4-loop and zero or more 3 -chains. The condition $c \geq-2$ shows that the number of 3 -chains is $0,1,2,3$ or 4 . In these cases we have $c=4,1,0,-1$ or -2 , and lemma 3.6 gives $v=4,1,2,1$ or 2 respectively. As claimed, $v=|c|$ unless $G=3+3+4 \ell$. For the optimality of opening a 4-loop when $G=$ (no, one, two or four 3-chains) we refer to lemma 3.6 .

Now suppose removing a 4-loop leaves the terminal bonus invariant. So $c\left(G-4_{\ell}\right)=c+4$. We first prove $v\left(G ; 4_{\ell}\right)=|c|$. By $c \geq-2$ we have $c\left(G-4_{\ell}\right) \geq 2$, so $v\left(G-4_{\ell}\right)=c\left(G-4_{\ell}\right)$. If $c=-2$ resp. -1 , then $c\left(G-4_{\ell}\right)=2$ resp. 3 , so $v\left(G-4_{\ell}\right)=2 \operatorname{resp} .3$, so $v\left(G ; 4_{\ell}\right)=2$ resp. 1 , which equals $|c|$. And if $c \geq 0$ then

$$
v\left(G ; 4_{\ell}\right)=\left|c\left(G-4_{\ell}\right)-4\right|=(c+4)-4=c=|c| .
$$

This completes the proof that $v\left(G ; 4_{\ell}\right)=|c|$.

If $c \geq 2$ then the optimality of opening a 4-loop is lemma 3.3(2). Otherwise, $v\left(G ; 4_{\ell}\right)=|c|=0,1$ or 2 by the previous paragraph. If $C$ is a component other than a 4-loop, then

$$
1 \leq v(G ; C) \underset{\bmod 2}{\equiv} v\left(G ; 4_{\ell}\right) \leq 2
$$

So $v(G ; C)$ cannot be less than $v\left(G ; 4_{\ell}\right)$. This proves the optimality of a 4-loop, hence $v(G)=v\left(G ; 4_{\ell}\right)=|c|$.

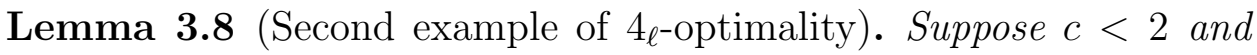
that $G \neq \emptyset$ has no 3-chains. If $G$ has a 4-loop then opening it is optimal. Regardless of whether $G$ has a 4-loop, if $c+4 f \geq 2$ then

$$
v=0,1,2,3,4 \text { in the cases } c \equiv 0, \pm 1, \pm 2, \pm 3,4 \bmod 8
$$

while if $c+4 f<2$ then

$$
v= \begin{cases}2 & \text { if } c \equiv 2 \bmod 4 \\ \text { otherwise: } & \\ (\text { whichever of } 0,1) \equiv c \bmod 2 & \text { if } f \text { is odd } \\ (\text { whichever of } 3,4) \equiv c \bmod 2 & \text { if } f \text { is even }\end{cases}
$$

Remark 3.9. In (3.5) we could replace every occurrence of $c$ by $\operatorname{size}(G)$, because the absence of 3-chains implies size $(G) \equiv c(G) \bmod 4$.

Proof. We begin with three special cases. First, suppose that $G$ is a union of 4-loops. Then $c+4 f=-4 f+4 f+8 \geq 2$, so we are asserting that $v(G)$ is given by (3.4). This is justified by lemma 3.6 .

Second, suppose $G$ has no 4 -loops. Then $c+4 f=c<2$, so we are asserting that $v(G)$ is given by (3.5). This is justified by lemma 3.4. 
Third, suppose $G$ has a 4 -loop and that $c=-2,-1,0$, or 1 . Then $c+4 f \geq 2$, so we are asserting that $v(G)$ is given by (3.4), namely $v(G)=2,1,0$, or 1 respectively. This is justified by lemma 3.7 .

For the general case we use induction on the number of components. So suppose that every position with fewer components than $G$, that satisfies the hypotheses of the lemma, also satisfies its conclusions. Regarding the cases already treated as base cases, we may suppose that $c(G)<-2$ and that $G$ has a 4-loop and a component other than a 4-loop. hence a longer loop or a chain of length $\geq 4$. The presence of this extra component shows that removing a 4-loop from $G$ does not change the terminal bonus. In particular, $c\left(G-4_{\ell}\right)=c+4<-2+4=2$. This and the minimality of $G$ show that $v\left(G-4_{\ell}\right)$ is given by whichever of (3.4) and (3.5) applies to $G-4_{\ell}$. To figure out which one applies we observe

$$
c\left(G-4_{\ell}\right)+4 f\left(G-4_{\ell}\right)=c(G)+4 f(G)
$$

This shows that whichever of (3.4) and (3.5) claims to describe $v(G)$ does indeed describe $v\left(G-4_{\ell}\right)$.

Our next step is to prove that $v\left(G ; 4_{\ell}\right)$ is equal to what the lemma claims is $v(G)$. That is, writing $w(\cdot)$ for the function on positions given by (3.4) and (3.5), we will prove $v\left(G ; 4_{\ell}\right)=w(G)$. Regardless of which of (3.4) and (3.5) applies to $G-4_{\ell}$, we have $v\left(G-4_{\ell}\right)=w\left(G-4_{\ell}\right) \in$ $\{0,1,2,3,4\}$. It follows that $v\left(G ; 4_{\ell}\right)=4-v\left(G-4_{\ell}\right)$. It is also easy to see that $w(G)=4-w\left(G-4_{\ell}\right)$ : for $(3.4)$ this uses the fact that $c(G)$ and $c\left(G-4_{\ell}\right)$ differ by $4 \bmod 8$, while for $(3.5)$ this uses the fact that $f(G)$ and $f\left(G-4_{\ell}\right)$ have different parities. It follows that $v\left(G ; 4_{\ell}\right)=w(G)$.

Now we prove the optimality of a 4-loop. Suppose for a contradiction that $C$ is a component of $G$ with $v(G ; C)<v\left(G ; 4_{\ell}\right)$. By

$$
2 \leq v(G ; C) \underset{\bmod 2}{\equiv} v\left(G ; 4_{\ell}\right) \leq 4
$$

we must have $v(G ; C)=2$ and $w(G)=v\left(G ; 4_{\ell}\right)=4$. The first of these forces $C$ to be a 4-chain with $v(G-4)=2$, or a 6 -loop with $v\left(G-6_{\ell}\right)=4$. Removing a $6_{\ell}$ leaves the terminal bonus invariant, while removing a 4 -chain increases it by 0 or 4 . In either case we have

$$
c(G-C) \leq c(G)+4<-2+4=2
$$

By induction, the lemma describes $v(G-C)$.

If $C$ is a 4-chain then $v(G-4)=2$ forces $c(G-4) \equiv 2 \bmod 4$, regardless of whether (3.4) or (3.5) applies to $G-4$. But then the absence of 3 -chains shows

$$
c(G) \equiv \operatorname{size}(G) \equiv \operatorname{size}(G-4) \equiv c(G-4) \equiv 2 \bmod 4
$$


This gives $w(G)=2$, regardless of whether 3.4 or 3.5 applies to $G$. This contradicts our early conclusion that $w(G)=4$. If $C$ is a 6-loop then $v\left(G-6_{\ell}\right)=4$ forces $c\left(G-6_{\ell}\right) \equiv 0 \bmod 4$, regardless of whether (3.4) or $(3.5)$ applies to $G-6_{\ell}$. This forces $c(G) \equiv 2 \bmod 4$, leading to the same contradiction.

We have proven the optimality of opening a 4-loop. So $v(G)=$ $v\left(G ; 4_{\ell}\right)=w(G)$ follows and the proof is complete.

The next two lemmas, taken together, are an analogue of sections 11-13 of [5]. But the formulations and arguments are quite different.

Lemma 3.10 (Third example of $4_{\ell^{-}}$optimality). Suppose $G$ has exactly one 3 -chain, and $\operatorname{size}(G) \equiv 3 \bmod 4$ and $c<2$. If $G$ has a 4-loop then opening it is optimal. Regardless of whether $G$ has a 4 -loop, if $c+4 f \geq 2$ then

$$
v(G)=1 \text { or } 3 \text { according to whether } c(G) \equiv \pm 1 \text { or } \pm 3 \bmod 8
$$

while if $c+4 f<2$ then

$$
v(G)=1 \text { or } 3 \text { according to whether } f \text { is odd or even. }
$$

Proof. This is similar in structure to the previous proof. Note that $c$ is odd because $G$ is. We begin with three special cases. First, if $G$ has no 4-loops then $c+4 f<2$, so the lemma claims $v=3$. This is justified by lemma 3.5. Second, if $G$ has a 4-loop and $c(G)= \pm 1$ then $c+4 f \geq 2$, so the lemma claims that $v=1$ and opening a 4-loop is optimal. This is justified by lemma 3.7. (The exception $3+3+3+4_{\ell}$ in that lemma is irrelevant because $\theta=1$.) Third, suppose that $G$ consists of a 3 -chain and $f>0$ many 4-loops. Then $c+4 f=5$, so the lemma asserts that $v(G)=1$ or 3 according to whether $c \equiv \pm 1$ or $\pm 3 \bmod 8$. The cases $c \equiv-1$ or $3 \bmod 8$ do not occur, and the cases $c \equiv 1$ or $-3 \bmod 8$ are equivalent to $f$ being odd or even, respectively. So we may quote lemma 3.6.

For the general case we induct on the number of components. So suppose every position with fewer components than $G$, that satisfies the hypotheses of the lemma, also satisfies its conclusions. Regarding the cases already treated as base cases, we may suppose that $c<-2$, and that $G$ contains a 4-loop and also a component that is neither a 3-chain nor a 4-loop. In particular, removing a 4-loop does not alter the terminal bonus. So $c(G-4 \ell)=4+c<2$. By induction, the lemma describes $v\left(G-4_{\ell}\right)$. In particular, $v\left(G-4_{\ell}\right)=1$ or 3 . These lead to $v\left(G ; 4_{\ell}\right)=3$ or 1 respectively.

Next we claim $v(G ; 3)=3$. We have $c(G-3) \leq c+4<2$, so lemma 3.8 computes $v(G-3)$. Which case of that lemma applies 
depends on the value of $c(G-3)+4 f(G-3)$. But both cases give

$$
v(G-3) \equiv \operatorname{size}(G-3) \equiv \operatorname{size}(G)-3 \equiv 0 \bmod 4
$$

So $v(G ; 3)=1+\mid(0$ or 4$)-2 \mid=3$. This proves the optimality of opening a 4-loop, because any component $C \neq 3,4_{\ell}$ has $v(G ; C)$ odd and at least 2 .

All that remains is to justify the stated value of $v(G)$. We know $v(G)=v\left(G ; 4_{\ell}\right)=4-v\left(G-4_{\ell}\right)$. On the other hand, the equality (3.6) from the previous proof also holds here, so that whichever of (3.7) and (3.8) purports to describe $v(G)$ does indeed describe $v\left(G-4_{\ell}\right)$. Examining these two formulas shows that $4-v\left(G-4_{\ell}\right)$ is the claimed value of $v(G)$. For $(3.7)$ this uses the fact that $c(G)$ and $c\left(G-4_{\ell}\right)$ differ by 4 , while for (3.8) this uses the fact that $f(G)$ and $f\left(G-4_{\ell}\right)$ have different parities.

Lemma 3.11 (Second example of 3-optimality). Suppose $c<-1$ and that either

(1) $\theta \geq 2$, or

(2) $\theta=1$ and $\operatorname{size}(G) \not \equiv 3 \bmod 4$.

Then opening a 3-chain is optimal, and $v=1$ or 2 according to whether $G$ is odd or even.

Proof. We begin by treating three special cases. First, if $G$ has no 4loops then we quote lemma 3.5. Second, if $G$ consists of 4 -loops and 3 -chains then we quote lemma 3.6.

Third, suppose that $G$ consists of a 3-chain and some loops. Then $G$ is odd, so our hypothesis on $\operatorname{size}(G)$ forces $\operatorname{size}(G) \equiv 1 \bmod 4$. Also $c(G-3)=3+c(G)<2$, so lemma 3.8 describes $v(G-3)$. Because $c(G-3) \equiv \operatorname{size}(G-3) \equiv 2 \bmod 4$, that lemma gives $v(G-3)=$ 2 , independently of whether (3.4) or $(3.5)$ applies to $G-3$. From $v(G-3)=2$ we get $v(G ; 3)=1$. Since $v(G)$ is odd and bounded above by $v(G ; 3)$, the 3 -chain is optimal and $v(G)=1$.

We have reduced to the case that $G$ has at least two chains, that it has a 4-loop, and that it has a component that is neither a 3-chain nor a 4-loop. It follows that the terminal bonus is invariant under the removal of either a 3-chain or a 4-loop. In particular, $c(G-3)=1+c<0$ and $c(G-4 \ell)=4+c<3$.

As a fourth special case, suppose $c=-2$. Then lemma 3.7 applies to both $G$ and $G-3$, giving $v(G)=2$ and $v(G-3)=1$. The first of these is our claimed value for $v(G)$, and the second shows $v(G ; 3)=2$. Since this equals $v(G)$, opening a 3 -chain is optimal. Henceforth we suppose $c(G)<-2$. 
Suppose $G$ is a counterexample with fewest possible components; we will derive a contradiction. The main step is to show $v(G ; 3) \in\{1,2\}$. Suppose first that $\theta=1$. Lemma 3.8 describes $v(G-3)$. Because $c(G-3) \equiv \operatorname{size}(G-3) \not \equiv 0 \bmod 4$, it says $v(G-3)=1,2$ or 3 . Then $v(G ; 3)=2,1$ or 2 respectively. Now suppose $\theta=2$ and $\operatorname{size}(G) \equiv 2$ mod 4. Then lemma 3.10 gives $v(G-3) \in\{1,3\}$, hence $v(G ; 3)=2$. Finally, suppose that either $\theta=2$ and $\operatorname{size}(G) \not \equiv 2 \bmod 4$, or that $\theta>2$. Then the minimality of $G$ shows that the current lemma describes $v(G-3)$. In particular, $v(G-3) \in\{1,2\}$, so $v(G ; 3) \in\{2,1\}$ also. This finishes the proof of $v(G ; 3) \in\{1,2\}$.

Since $G$ is a counterexample, it has a component $C$ with $v(G ; C)<$ $v(G ; 3)$. This is obviously impossible if $v(G ; 3)=1$, so we must have $v(G ; 3)=2$ and $v(G ; C)=0$. The latter forces $C$ to be a 4-loop with $v\left(G-4_{\ell}\right)=4$. If $c\left(G-4_{\ell}\right)<-1$ then induction would give the contradiction $v\left(G-4_{\ell}\right) \in\{1,2\}$. Therefore $c\left(G-4_{\ell}\right) \geq-1$. On the other hand, $c\left(G-4_{\ell}\right)=c+4<2$, so we must have $c\left(G-4_{\ell}\right)=-1,0$ or 1 . There must be no 4 -loops in $G-4_{\ell}$, or else lemma 3.7 would give the contradiction $v\left(G-4_{\ell}\right)=\left|c\left(G-4_{\ell}\right)\right|=(0$ or 1$) \neq 4$. But then lemma 3.5 gives the contradiction $v\left(G-4_{\ell}\right) \in\{1,2,3\}$.

Proof of theorem 1.1. This amounts to combining the results above. First suppose $c \geq 2$. If $G=3+$ (one or more loops) then theorem 3.2 shows that opening the shortest loop is optimal. Otherwise, the same theorem shows that the standard move (opening a 3-chain) is optimal.

Henceforth we may assume $c<2$. If $G$ has no 3-chains or 4-loops then we must prove that the standard move is optimal. Lemma 3.4 shows that $G$ has a 6-loop, and that opening it (which is the standard move) is optimal. If $G$ has a 3 -chain but no 4 -loops then we must prove that the standard move (opening a 3-chain) is optimal. This is part of lemma 3.5. If $G=4_{\ell}+3+3+3$ then again we must prove that the standard move (opening a 3-chain) is optimal. This is part of lemma 3.6 .

We have reduced to the case that $c<2$ and that $G \neq 4_{\ell}+3+3+3$ has a 4-loop. If $c \in\{0, \pm 1\}$ then lemma 3.7 shows that opening a 4-loop is optimal.

Finally, suppose $c \leq-2$. If $G$ has no 3 -chains then we must prove that the standard move (opening a 4-loop) is optimal. This is part of lemma 3.8. The only remaining cases are $G=3+4_{\ell}+H$. If $H$ has no 3 -chains and $4 \mid \operatorname{size}(H)$ then we must show that opening a 4-loop is optimal, which is part of lemma 3.10. On the other hand, if $H$ has a 3 -chain or $4 \nmid \operatorname{size}(H)$ then we must show that the standard move (opening the 3 -chain) is optimal. This is part of lemma 3.11 . 


\section{Game values and CONTROller strategy}

The controller's strategy in theorem 1.3 depends on being able to recognize when a position $G$ has value $>2$. We will give two ways to compute the value. First we give an explicit value in terms of $c(G)$, $f(G)$ and the overall size and shape of $G$. This is complicated. Then we give our preferred method, whose essential case computes $v(G)$ by starting with $c\left(G_{0}\right)$ for a certain smaller position $G_{0}$, and then applying a simple process. The following result immediately implies theorem 1.4 .

Theorem 4.1 (Values - explicit). Suppose $G$ is a nonempty Dots 8 Boxes position consisting of loops and long chains. Then its value $v=$ $v(G)$ is given by the first of the following cases that applies:

(1) If $c \geq 2$ then $v=c$.

(2) If $c=0$ and $G=4_{\ell}+$ (anything except $\left.3+3\right)$, then $v=0$.

(3) If $\theta=0$, or if $\theta=1$ and $\operatorname{size}(G) \equiv 3 \bmod 4$, then

(a) if $c+4 f \geq 2$ then $v=0,1,2,3,4$ according to whether $c \equiv 0, \pm 1, \pm 2, \pm 3,4 \bmod 8$.

(b) if $c+4 f<2$ then

(i) if $G$ is odd then $v=1$ resp. 3 if $f$ is odd resp. even.

(ii) if $\operatorname{size}(G) \equiv 2 \bmod 4$ then $v=2$.

(iii) if $\operatorname{size}(G) \equiv 0$ mod 4 then $v=0$ resp. 4 if $f$ is odd resp. even.

(4) In all other cases, $v=1,2$ as $G$ is odd,even.

Proof. (1) If $c \geq 2$ then $v=c$ by theorem 3.2. So suppose $c<2$.

(2) If $c=0$ and $f>0$ and $G \neq 3+3+4_{\ell}$ then lemma 3.7 gives $v=0$, as we are asserting.

(3) Now suppose $\theta=0$, or that $\theta=1$ and $\operatorname{size}(G) \equiv 3 \bmod 4$. If $c+4 f \geq 2$ then the theorem's value for $v$ is justified by lemma 3.8 (if $\theta=0$ ) or 3.10 (if $\theta=1$ ). The $c+4 f<2$ case is also justified by these lemmas.

(4) In all remaining cases we have $\theta \geq 2$, or $\theta=1$ and $\operatorname{size}(G) \not \equiv 3$ mod 4. We must prove $v(G) \in\{1,2\}$. If $G$ has no 4 -loop then we refer to lemma 3.5. So we suppose $f>0$. If $c= \pm 1$ then $v=1$ by lemma 3.7 . If $c=0$ then we must have $G=4_{\ell}+3+3$, or else case (2) would have applied. This special case has value 2 by lemma 3.6. Finally, if $c<-1$ then we refer to lemma 3.11 .

There is a way to replace the complicated case (3) by a simpler iterative procedure. In practice it is easier and less error-prone than working through the tree of subcases. It is quicker to use than explain, so the reader might want to look ahead at example 4.3. We will define a union $G_{0}$ of components of $G$. The strategy for computing $v(G)$ is 
to show $v\left(G_{0}\right)=c\left(G_{0}\right)$ and that $v(G)$ is got from $v\left(G_{0}\right)$ by a simple process.

Suppose $\theta \leq 1$. We define the core $G_{0}$ of $G$ as the union of the following components of $G$. Its loops are all the loops of length $\geq 8$. Its chains are either all the chains of length $\geq 4$ (if there are any) or the 3-chain (if $G$ has one and no other chains). The core has the useful property that if $H$ is the union of $G_{0}$ and possibly some more components of $G$, then $H_{0}=G_{0}$. Write $\theta^{\prime} \in\{0,1\}$ for the number of 3-chains in $G-G_{0}$.

We define maps $\mathbb{Z} \rightarrow \mathbb{Z}$ :

$$
\Sigma(x)=|x-4|+2 \quad \Theta(x)=x-1 \quad \Phi(x)=|x-4|
$$

Only their values on $\mathbb{Z}_{\geq 0}$ will be important. We think of $\Sigma$ as decreasing any $x \geq 2$ in steps of size 2 until $x$ enters $[2,4]$, where $\Sigma$ acts as reflection across 3. And $\Phi$ decreases $x$ in steps of size 4 until $x$ enters $[0,4]$, where $\Phi$ acts as reflection across 2 . These operations are from [5], but the generality in which we use them is new.

Theorem 4.2 (Values-procedural). Suppose $G$ is a nonempty Dots ES Boxes position consisting of loops and long chains. Then

(1) if $c \geq 2$ then $v=c$;

(2) if $c=0$, and $G$ has a 4-loop, and $G \neq 4_{\ell}+3+3$, then $v=0$;

(3) if $\theta=0$, or if $\theta=1$ and $\operatorname{size}(G) \equiv 3 \bmod 4$, then

$$
v=\Phi^{f} \Theta^{\theta^{\prime}} \Sigma^{s}\left(c\left(G_{0}\right)\right)
$$

In all other cases $v=1$ or 2 according to the parity of $G$.

Example 4.3. Say $G=\left(8_{\ell}^{2}+18\right)+6_{\ell}^{9}+3+4_{\ell}^{101}$, where the parentheses indicate $G_{0}$. Because $\theta=1$ we check that $\operatorname{size}(G) \equiv 3 \bmod 4$, so the theorem applies. We begin with $v\left(G_{0}\right)=c\left(G_{0}\right)=18$. Adjoining the first seven 6-loops decreases this by 2 each time, leaving 4 . Adjoining the remaining two 6-loops bounces this to 2 and back to 4 (reflecting across 3). Adjoining the 3-chain reduces this to 3. Adjoining the 4loops bounces this between 3 and 1 an odd number of times, leaving $v(G)=1$.

Proof. Unlike theorem 4.1, no case (1)-(3) has priority over any other. When two apply then we are asserting that both are correct. Cases (1) and (2) are justified by theorem 3.2 and lemma 3.7 respectively. The "in all other cases" argument is the same as for theorem 4.1.

For case (3) we first prove $v\left(G_{0}\right)=c\left(G_{0}\right)$. If $G_{0}=\emptyset$ then both sides are 0 , so suppose $G_{0} \neq \emptyset$. Every component of $G_{0}$ is a loop of length $\geq 8$ or a chain of length $\geq 4$, or a 3 -chain. There is at most one 3 -chain. Every term in the formula (2.3) for $c\left(G_{0}\right)$ is nonegative, 
except that one term can be -1 . So $c\left(G_{0}\right) \geq \operatorname{tb}\left(G_{0}\right)-1 \geq 3$, which implies $v\left(G_{0}\right)=c\left(G_{0}\right)$.

Next we observe that the initial moves in an optimal strategy for the opener is to open all the 4-loops, then the 3-chain (if $G-G_{0}$ has one), then the 6-loops. This follows from theorem 1.1. Therefore $v(G)$ can be got by working backwards from $v\left(G_{0}\right)=c\left(G_{0}\right)$. We defined $\Sigma$ so that $v(H)=\Sigma\left(v\left(H-6_{\ell}\right)\right)$ for any position $H$ in which opening a 6-loop is optimal. It follows that if $G-G_{0}$ has no 3-chain or 4-loops, then $v(G)=\Sigma^{s}\left(v\left(G_{0}\right)\right)$. If $G-G_{0}$ has a 3 -chain but no 4-loops, then instead we use $v(G)=1+|v(G-3)-2|=\Theta(v(G-3))$. The second inequality here comes from the fact that $G_{0} \subseteq G-3$ is nonempty (else the 3-chain would lie in $G_{0}$ ) without 3 -chains or 4 -loops, hence has value $\geq 2$ by lemma 3.4. We have proven $v=\Theta^{\theta^{\prime}} \Sigma^{s}\left(c_{0}(G)\right)$. For general $G$ the same argument shows

$$
v(G)=\Phi^{f}(v(G)-(\text { all 4-loops }))=\Phi^{f} \Theta^{\theta^{\prime}} \Sigma^{s}\left(v\left(G_{0}\right)\right) .
$$

\section{Consequences for midgame Play}

This section discusses how to use our results in actual play. After reaching a position consisting of loops and long chains, one can play optimally by following the strategies in the introduction. But before play gets that far, there are opportunities to influence the shape of the endgame.

We write from the perspective of a player ("you") who expects to be the opener once the game settles down to a union of loops and long chains. This means that you expect to lose the Nimstring game. Assuming you are right in this expectation, your only chance of victory is to gain a large enough advantage in captured boxes before the endgame. For a line of play under consideration, we write $G$ for the resulting endgame and $A$ for your advantage in boxes at the time play reaches that position. Players prefer odd $\times$ odd boards because ties are impossible, so we restrict to this case. As a consequence, $A$ and $\operatorname{size}(G)$ have different parities. We also assume that you will create enough 3chains and 4- and 6-loops so that $c(G)<2$ and hence $v(G) \in\{0, \ldots, 4\}$.

A simple rule of thumb is: if $A \leq 1$ then you will lose, while if $A \geq 2$ then you will win. This is not really true, but it is true "generically" in the sense that theorem 4.1 shows that almost all positions with $c(G)<2$ have value 1 or 2 . If $A=1$ then the only way you can win is to arrange for $v(G)=0$. If $A=2$ resp. 3 then the only way you can lose is for $v(G)=3$ resp. 4 . According to theorem 4.1, each of these imposes very strong constraints on $G$. We now consider in more detail the possibilities for pairs $(A, G)$. Your goal, for given $A$, is to steer 
the game toward an endgame $G$ satisfying $A-v(G)>0$. We refer to theorem 4.1 throughout the analysis. Any $(A, G)$ with $A \leq 0$ is lost, while any $(A, G)$ with $A \geq 4$ is a win.

First consider $(A, G)$ with $A=1$. You have probably lost, but if you can arrange $v(G)=0$ then you will win. Your opponent will try to block the creation of a 4-loop, and if she succeeds then you will lose $(f=0$ implies $v \neq 0$, hence $v \geq 2)$. So we assume that you can arrange for a 4-loop to be present. You will win if you can also arrange for $c=0$, with the single exception $G=4_{\ell}+3+3$.

Our advice past this point is less likely to be useful because it gets into the complicated case (3) of theorem 4.1. If you cannot arrange for $c=0$, then you must arrange for $\theta=0$ and $\operatorname{size}(G) \equiv 0 \bmod 4$, so we assume you can achieve this. (By $c \neq 0, \theta>0$ implies $v>0$. And if $\theta=0$ then $v=0$ forces $\operatorname{size}(G) \equiv 0 \bmod 4$.) You will win if you can also arrange for the number $s$ of 6 -loops to be small and $c \equiv 0$ mod 8 , or if you can arrange for $s$ to be large and $f$ to be even. Here and below we write " $s$ small" and " $s$ large" as stand-ins for the more precise statements $c+4 f \geq 2$ and $c+4 f<2$ respectively. (Because $\theta=0$, making $c+4 f$ small requires 6-loops.) Actual play is unlikely to lead to many 6-loops, so one could ignore the " $s$ large" case without much loss.

Now consider $(A, G)$ with $A=2$. You will probably win, but if your opponent can arrange for $v(G)=3$ then you will lose. If you can create two 3-chains then you will win. Even a single 3-chain will do if $\operatorname{size}(G) \not \equiv 3 \bmod 4$. If you cannot acheive this, then you must arrange for both of the second pair of alternatives of theorem 1.4 to fail. That is, you must arrange for $s$ to be small and $c(G) \equiv \pm 1 \bmod 8$, or for $s$ to be large and $f$ to be odd.

Finally consider $(A, G)$ with $A=3$. This is similar to the $A=2$ case but easier since you will win unless $v(G)=4$. If you arrange for $G$ to have a 3 -chain, or $\operatorname{size}(G) \equiv 2 \bmod 4$, then you will win. (This uses the evenness of $G$.) Suppose you cannot acheive either of these, so $\theta=0$ and $\operatorname{size}(G) \equiv 0 \bmod 4$. Then you must aim for $s$ to be small and $c(G) \equiv 0 \bmod 8$, or for $s$ to be large and $f$ to be odd.

\section{REFERENCES}

[1] E. Berlekamp, The Dots and Boxes game, A K Peters 2000.

[2] E. Berlekamp and K. Scott, Forcing your opponent to stay in control of a loony dots-and-boxes endgame, in More Games of No Chance (Berkeley, CA, 2000), 317-330 in vol. 42 of MSRI Publ., Cambridge U. Press, 2002.

[3] E. R. Berlekamp, J. H. Conway and R. K. Guy, Winning Ways for your mathematical plays, vol. 2, Academic Press, 1982. 
[4] E. Berlekamp and D. Wolfe, Mathematical Go Endgames: Nightmares for the Professional Go Player, Ishi Press, 1994.

[5] Kevin Buzzard and Michael Ciere, Playing simple loony Dots and Boxes endgames optimally, arxiv:1305.2156

Department of Mathematics, University of Texas, Austin

E-mail address: allcock@math.utexas.edu

URL: http://www.math.utexas.edu/ ${ }^{\sim}$ allcock 